\title{
Measured effects of polymer additives on turbulent-velocity fluctuations at various length scales
}

\author{
P. Tong \\ Department of Physics, Oklahoma State University, Stillwater, Oklahoma 74078 \\ W. I. Goldburg \\ Department of Physics and Astronomy, University of Pittsburgh, Pittsburgh, Pennsylvania 15260 \\ J. S. Huang \\ Exxon Research and Engineering Company, Annandale, New Jersey 08801
}

(Received 30 September 1991)

\begin{abstract}
Turbulent-drag reduction in a dilute polymer solution has been studied using the technique of photon-correlation homodyne spectroscopy to measure velocity differences in a concentric cylinder cell, in which the inner cylinder rotates. A large anisotropic suppression of turbulent-velocity differences is found in the bulk region of the turbulent fluid. The suppression effect occurs at various length scales up to $\approx 1 \mathrm{~mm}$, which is far beyond the Kolmogorov dissipation length $l_{d}(\approx 0.04 \mathrm{~mm})$. The large-scale velocity fluctuations are suppressed, but their statistical properties over varying length scales remain unchanged. The small-scale fluctuations, on the other hand, are damped out much more strongly, resulting in a different functional form for the velocity-density function. The latter observation is consistent with the notion that the polymer-turbulence interaction causes a truncation of the turbulent-energy cascade at small scales. The measurements are also in agreement with laser Doppler velocimetry results that in turbulent polymer solutions the lifetime of large-scale vortices is increased, and the high-frequency velocity fluctuations (small-scale motions) are reduced.

PACS number(s): 47.50. $+\mathrm{d}, 47.25 .-\mathrm{c}, 42.25 .-\mathrm{p}, 61.25 .-\mathrm{f}$
\end{abstract}

\section{INTRODUCTION}

It has been known that trace amounts of flexible polymers in solution can significantly reduce the drag of turbulent flow below that for the solvent alone. The phenomenon, which has stimulated much research in past decades [1-4] has important applications, such as increasing pipeline capacities and speeding ships. However, the exact mechanism for the drag-reduction effect is not well understood, and only tentative explanations exist. It is believed that turbulent flow strongly stretches polymer chains in the solution, and the reaction of the stretched polymer chains to the flow gives rise to the drag reduction. One hypothesis for the drag reduction assumes that the stretched polymer chains affect the turbulent-energy cascade at small scales via the enhancement of the elongational viscosity $[2,5]$ or via some elastic modulus of the stretched chains [6]. Another hypothesis assumes that polymer molecules cause stabilization of viscous boundary layers, and hence reduce the production of turbulence $[3,7,8]$.

Two important questions concerning the phenomenon of turbulent-drag reduction are (1) whether the influence of polymer additives is restricted to regions near the wall or if it also extends to the turbulent core region and (2) at what length scales polymer additives affect the turbulent flow. Is the turbulence damped at small scales comparable to the turbulent dissipation length or at both the small and large scales? To answer these questions, one has to study spatial structures of the turbulence in polymer solutions at different positions and at various length scales. Measurements of the local velocity $v(r(t))$ by laser Doppler velocimetry (LDV) $[9,10]$, which was used in most previous experiments $[1,3,11-13]$, provided useful information about the effect of polymer additives on vortex structures of the turbulence. However, the "frozen-turbulence" assumption [14] must be invoked to relate the measured temporal signals with spatial structures of the turbulence.

For strongly turbulent flow, as in the case of turbulent-drag reduction, it is more interesting to measure instantaneous velocity difference $\mathbf{V}(\mathbf{R}, t)$ over a distance $\mathbf{R}$, where

$$
\mathbf{V}(\mathbf{R}, t)=\mathbf{v}(\mathbf{r}(t))-\mathbf{v}(\mathbf{r}(t)+\mathbf{R}) .
$$

In this paper we report an experimental study of turbulent-drag reduction using the technique of photoncorrelation homodyne spectroscopy (HS) [15] from which the probability-density function $P(V, R)$ of the relative velocity $V(R, t)$ can be inferred [16-18]. The experiment reveals that polymer additives cause suppression of velocity fluctuations over the whole spectrum of length scales. The large-scale velocity fluctuations are suppressed, but their statistical properties over varying length scales remain unchanged. The small-scale fluctuations, on the other hand, are damped out much more strongly, resulting in a different functional form for the density function $P(V, R)$. The suppression of velocity fluctuations at small length scales is found in the bulk region of the turbulent fluid.

With the HS scheme, the scattering is produced by 
small seed particles in the fluid, which follow the local flow. The photodetector records the scattered light, which is the beating of Doppler-shifted light scattered by pairs of flowing particles. The output of the detector is therefore modulated at frequencies equal to the differences in Doppler shifts of all particle pairs in the scattering volume. For each particle pair separated by a distance $\mathbf{R}$, this difference is $\mathbf{q} \cdot \mathbf{V}(\mathbf{R}, t)$, with the scattering vector $\mathbf{q}$ having the amplitude $q=(4 \pi n / \lambda) \sin (\theta / 2)$. Here $\theta$ is the scattering angle, $n$ is the refractive index of the fluid, and $\lambda$ is the wavelength of the incident light. With the so-called homodyne method, one measures the intensity autocorrelation function [15]

$g(t)=\left\langle I\left(t^{\prime}+t\right) I\left(t^{\prime}\right)\right\rangle=\left\langle I\left(t^{\prime}\right)\right\rangle^{2}[1+b G(q t, L)]$,

where $I(t)$ is the intensity of the scattered light and the angle brackets represent a time average over $t^{\prime}$. In the last equality, $b$ is a constant which depends on the geometry of the experimental setup.

It has been shown $[16,17]$ that the function $G(q t, L)$ in Eq. (1) has the form

$G(q t, L)=\int_{0}^{L} d R h(R) \int_{-\infty}^{\infty} d V P(V, R) \cos (q t V)$,

where $V(R, t)$ is the component of $\mathbf{V}(R, t)$ along the scattering vector q. In the above, $h(R)=2(1-R / L) / L$ is the number fraction of particle pairs separated by a distance $R$ in the scattering volume. The scattering volume viewed by a photodetector is assumed to be quasi-onedimensional with length $L$. Equation (2) states that the light scattered by each pair of particles contributes a phase factor $\cos [\mathbf{q} \cdot \mathbf{V}(\mathbf{R}) t]$ (due to frequency beating) to the intensity correlation function $G(q t, L)$, and $G(q t, L)$ is an incoherent sum of these ensemble averaged (or time averaged) phase factors over all the particle pairs in the scattering volume. The ensemble average of the phase factor, $\cos [\mathbf{q} \cdot \mathbf{V}(\mathbf{R}) t]$, involves the velocitydensity function $P(V, R)$. The weighted average over $R$ is required because the photodetector receives light from particle pairs having a range of separations $(0<R<L)$, and their contributions to the scattering intensity $I(t)$ are proportional to the number fraction of the particle pairs in the scattering volume. The function $G(q t, L)$ yields information about the velocity differences in the $q$ direction and at various scales $R$ (eddy sizes) up to $L$.

In the previous paper [19] (hereafter referred to as I) the present authors have utilized the HS technique to measure turbulent-velocity differences in a concentric cylinder cell in which the inner cylinder rotates. Different components of the velocity differences were probed by changing the direction of the scattering vector q. Velocity differences at various length scales were measured by varying the size of the scattering volume. The measurements of the correlation function $G(q t, L)$ reveal that the turbulence in the flow cell is neither homogeneous nor isotropic. Nevertheless, the measured $G(q t, L)$ is found to be of the scaling form

$$
G(q t, L)=G(\kappa),
$$

where $\kappa=q t u(L)$, with $u(L) \sim L^{\xi}$ being the characteristic turbulent velocity at the length scale $L$. The scaling be- havior of $G(q t, L)$ was observed at various observation points and for various directions of $q$. The exponent $\xi$ varies with scattering geometries and with the type of cell used (one cell contained radial fins and the other did not). The above scaling properties of $G(q t, L)$ were observed over various length scales $(L)$ and Reynolds numbers when the spatial position of the laser beam and the scattering geometry are fixed. However, when one compares the correlation functions measured at different scattering geometries or at different spatial positions (keeping the other conditions unchanged), it is found that these functions do not scale mutually.

A scaling $G(q t u(L))$ implies that the velocity-density function $P(V, R)$ has a scaling form $[16,20]$

$$
P(V, R)=Q(V / u(R)) / u(R),
$$

provided $u(R) \sim R^{\xi}$. This can be shown if one substitutes Eq. (4) into Eq. (2), and $G(q t, L)$ in Eq. (2) becomes [16]

$$
G(q t u(L))=\int_{0}^{L} d R h(R) F(q t u(R)),
$$

where $F(x)$ is the Fourier cosine transform of $Q(V / u(R))$. The above form for $P(V, R)$ implies that the turbulent velocity $V(R, t)$ is scale invariant, i.e., all its moments $\left\langle V(R, t)^{n}\right\rangle$ obey a power law in $R$ [16]. This is expected from the Kolmogorov theory of isotropic turbulence [ $21-23]$. The above scaling results have been observed for turbulent flows in a pipe [24] and in a square tunnel [25] at moderate Reynolds numbers. Our measurements in the Couette cell, therefore, suggest that the scaling argument can also be used to describe turbulent flows lacking isotropy and homogeneity. This is in agreement with the recent theoretical calculation by Knight and Sirovich [26], which showed that the above scaling arguments for homogeneous and isotropic turbulence are still valid for certain inhomogeneous turbulent flows. Our experiment also suggests that the exact functional form of the velocity-density function $P(V, R)$ (which is of scaling form) may vary with spatial positions and orientations in turbulent flows, reflecting the inhomogeneity and anisotropy of the turbulence.

The present paper reports measurements of $G(q t, L)$ made in a flow cell identical to that in $I$, but with water replaced by a polymer solution. This experiment differs from most previous experimental studies [1,3,11], which have focused on the straight-pipe system due to its obvious applications. The scale-invariant feature of the relative velocity fluctuations seen in water is also observed in the polymer solution. By comparing the measurements in the polymer solution with those in water, we find a large anisotropic suppression of turbulent-velocity differences in the bulk region of the turbulent fluid. The suppression effect occurs at various length scales $R$ up to $L \approx 1 \mathrm{~mm}$, which is far beyond the Kolmogorov dissipation length $l_{d}(\approx 0.04 \mathrm{~mm})$. The suppression of velocity differences is associated with an enhancement of the turbulent-drag reduction. The experiment reveals that the predominant polymer effect is on the velocity component along the mean flow direction. Gradients of this component appear to be suppressed in all directions in 
which the gradients are substantial.

Section II of the paper contains experimental details, including a brief description of the experimental methods and the sample characterizations. The results are given in Sec. III and are discussed in Sec. IV. Finally, the work is summarized in Sec. V.

\section{APPARATUS AND METHODS}

The flow cell and the experimental setup are the same as that shown in Fig. 1(a) of I. Refer to Fig. 1(a) and associated text in $I$ for a description of the experimental setup. The outer cylinder of the flow cell was made of Plexiglass to admit the incident light and observe the scattering. It was $12.7 \mathrm{~cm}$ both in height and in diameter. The inner rotating cylinder was a smooth brass tube, $9.4 \mathrm{~cm}$ in height and $5.7 \mathrm{~cm}$ in diameter. The tube was shafted along its axis and was mounted on the vertical axis of the outer cylinder through bearings. There was a filling stem on the top lid of the cylinder, and air bubbles could leave from the stem. The flow cell was mounted on the center of a square water bath, which was fixed on an optical table. The water bath was used to index match the cylindrical surface of the flow cell for the scattering measurements.

The flow cell was filled with a liquid (water or a polymer solution) seeded with small polystyrene latex spheres of diameter $0.1 \mu \mathrm{m}$. These particles follow the local flow [27] and scatter light. The volume fraction of the seed particles was $\simeq 10^{-4}$. At this particle concentration, the particle mean spacing is much larger than their diameter (dilute solution) but much smaller than the smallest turbulent scale (sufficient sampling). For the particlepolymer mixture the scattering was predominantly from the seed particles, because the polymer molecules scatter light very weakly.

Measurements of the autocorrelation function $g(t)$ were performed with a standard light-scattering apparatus and a multichannel correlator (Brookhaven Instruments Model No. BI-2030AT). The scattering geometries used in the experiment are sketched in Fig. 1. The direction of the incident beam was varied in three directions: the azimuthal $(\phi)$ direction (A in Fig. 1), and the radial ( $r$ ) direction ( $R$ in Fig. 1), and the vertical $(z)$ direction. In the latter case we employed two scattering geometries by changing the incident position of the vertical beam in the cell $\left[\mathrm{V}_{a}\right.$ (vertical azimuthal) and $\mathrm{V}_{r}$ (vertical radial) in Fig. 1]. The incident beam $\left(q_{i}\right)$ and the aligned optical apparatus $\left(\mathrm{L}_{2}, \mathrm{~S}\right.$, and $\left.\mathbf{P M}\right)\left(\mathrm{q}_{s}\right)$ define a scattering plane. In the $\mathrm{V}_{a}$ geometry the normal direction of the scattering plane is perpendicular to the mean flow direction, and in the $\mathrm{V}_{r}$ geometry the normal direction of the scattering plane is parallel to the mean flow direction (see Sec. III B for discussions about the baffled cell in the two geometries). For the four scattering geometries, the corresponding scattering vectors $\mathbf{q}=\mathbf{q}_{i}-\mathbf{q}_{s}$ are

$$
\begin{array}{ll}
\mathbf{q}_{\mathrm{A}}=q_{\phi} \mathbf{e}_{\phi}+q_{r} \mathbf{e}_{r}, & \mathbf{q}_{\mathbf{R}}=q_{\phi} \mathbf{e}_{\phi}+q_{r} \mathbf{e}_{r}, \\
\mathbf{q}_{\mathbf{v}_{a}}=q_{z} \mathbf{e}_{z}+q_{\phi} \mathbf{e}_{\phi}, & \mathbf{q}_{\mathbf{v}_{r}}=q_{z} \mathbf{e}_{z}+q_{r} \mathbf{e}_{r} .
\end{array}
$$

In the above, the $\mathbf{e}_{j}$ are unit vectors. One can probe

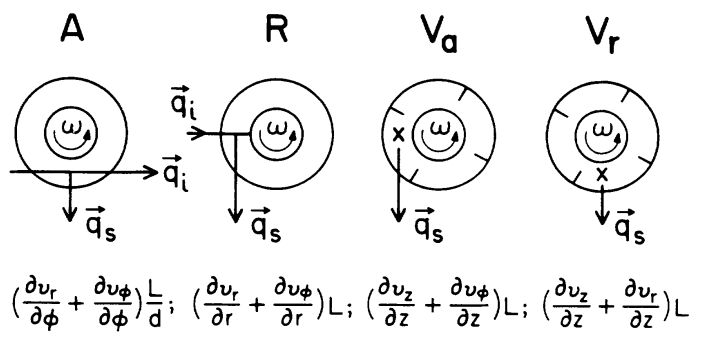

FIG. 1. Top view of flow cells and the scattering geometries ( $z$ axis is perpendicular to the paper): $\mathbf{q}_{i}$, incident wave vector; $\mathbf{q}_{s}$, scattering wave vector; $X$, incident position of the vertical laser beam. The components of velocity differences probed in each geometry are also listed, where $d$ is the distance between the laser beam and the rotation axis, and $L$ is the length of the laser beam viewed by the photodetector. The $A$ and $R$ geometries were also used in studies involving the baffled cell. [A schematic of the experimental setup appears in Fig. 1(a) of I.]

different components of the velocity difference $\mathbf{V}(\mathbf{R}, t)$ by varying the direction of $\mathbf{q}$, since the correlation function $G(q t, L)$ in Eq. (2) is only sensitive to the product $\mathbf{q} \cdot \mathbf{V}(\mathbf{R}, t)$. Figure 1 lists the components of the velocity gradients measured in each geometry. Notice that $L$ is the length of the laser beam viewed by the photomultiplier, and it can be varied by opening the jaws of the slit.

The polymer used in this study was polyethylene oxide (PEO), a commonly used water-soluble drag-reducing agent. The polymer powder was first dissolved in distilled water at the concentration $c \simeq 2.5 \times 10^{-3} \mathrm{~g} / \mathrm{cm}^{3}$, which was five times larger than the polymer overlap concentration $c^{*} \simeq 0.5 \times 10^{-3} \mathrm{~g} / \mathrm{cm}^{3}$. Next, a known amount of the high-concentration solution was mixed with distilled water at a desired concentration. The solution was kept for $24 \mathrm{~h}$ prior to use to allow for full dissolution. We always used fresh samples (less than a week old) to avoid possible chemical degradation. The nominal molecular weight $M_{w}$ of the PEO polymer was $5 \times 10^{6}$, as determined by a rheological measurement [28]. The viscosity of the polymer solution was measured by a Contravases low-shear Couette viscometer. The measured viscosity $\eta$ of the PEO solution at the concentration $c=100 \mathrm{ppm}$ (parts per million by weight) was $1.16 \eta_{w}$, where $\eta_{w}=0.01 \mathrm{P}$ is the viscosity of water. For the 50ppm PEO solution, the measured viscosity increment was reduced by half $\left(\eta / \eta_{w}=1.08\right)$. Up to the shear rate 100 $\mathrm{sec}^{-1}$, the measured viscosities were independent of the shear rate.

Using static and dynamic-light-scattering techniques [15], we measured the radius of gyration, $R_{g}$, and the hydrodynamic radius $R_{h}$ of the polymer molecules. Figure 2 presents the scattering data from the PEO aqueous solution with the concentration $c=272 \mathrm{ppm}$. The data are plotted as $I_{0} /\left[I(q)-I_{0}\right]$ versus $q^{2}$, where $I(q)$ is the scattering intensity of the polymer solution measured at the scattering angle $\theta$ (or, equivalently, at the scattering vector $q$ ) and $I_{0}$ is the light intensity scattered from the solvent alone. From the slope of the fitted straight line, we obtain the radius of gyration $R_{g}=160 \mathrm{~nm}$. The dynamic-light-scattering measurements give $R_{h} \simeq 80 \mathrm{~nm}$. 


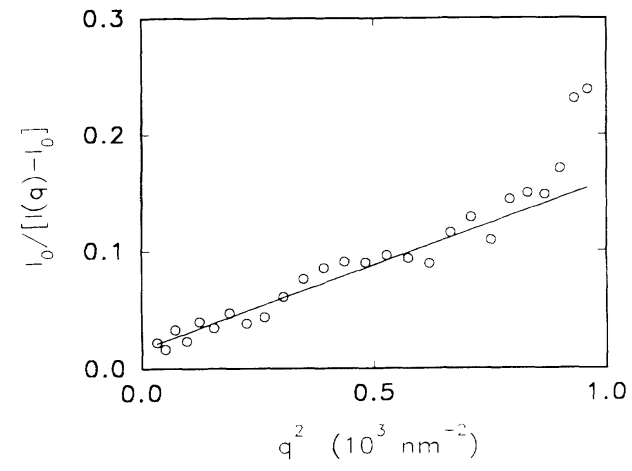

FIG. 2. The plot of $I_{0} /\left(I(q)-I_{0}\right)$ vs $q^{2}$ for the PEO solution with the concentration $c=272 \mathrm{ppm}$. The solid line is a linear fit to the data points up to $q^{2}=0.75 \times 10^{-3} \mathrm{~nm}^{-2}$.

Because of the polydispersity of the polymer sample, the measured value of $R_{h}$ shows some $q$ dependence, and the above value was obtained at $\theta=90^{\circ}$. The measured viscosity and the radius of gyration $R_{g}$ for the PEO polymer are both very close to the tabulated values for single polymer chains [29]. This suggests that our polymer solution is indeed a dilute solution $\left(c<c^{*}\right)$.

The turbulent drag in the flow cell was determined by measuring the wall stress $T_{w}$ (force per unit area) on the inner rotating cylinder. A torque-sensitive permanentmagnet dc motor was used to drive the inner rotating cylinder. The rotating speed of the motor was varied by changing the input voltage of the motor through a dcmotor controller. The driving current was directly measured using a $6 \frac{1}{2}$-digit voltmeter. For this type of motor the driving current is proportional to the torque exerted on the rotating cylinder. The proportionality constant (the torque constant) was obtained by running the motor as a generator (driven by another motor) and measuring the generated voltage as a function of the rotating speed. The rotating speed of the motor was measured by an electronic stroboscope.

An important question in the study of turbulence concerns the velocity fluctuations at different length scales. Understanding the polymer-turbulence interaction at various length scales is of fundamental interest, since many theoretical models have suggested that polymer molecules affect the turbulent-energy cascade at small scales near the dissipation length $l_{d}$. In Eq. (5) we have shown that the function $G(q t, L)$ senses fluid motions from the largest scale $L$, which is controlled by a slit, down to the smallest scale present in the flow. Since the injection rate of the turbulent energy is finite, $F(q t u(R))$ must be a decaying function [30] and the decay rate of $F(q t u(R))$ is proportional to $q u(R)$, as can be shown by a simple dimensional argument [16]. Equation (5) thus states that $G(q t, L)$ is a weighted sum of decaying functions, each of them characterizing the fluid motion at the length scale $R$, which is in the range between $L$ and the smallest scale of the turbulent flow. For the sake of the argument, let us imagine that there were only two modes in a turbulent flow: one was at the scale $L$ and the other at the Kolmogorov scale $l_{d}$, which is much smaller than $L$. The function $G(q t, L)$ is then a sum of $F(q t u(L))$ and
$F\left(q t u\left(l_{d}\right)\right)$ (assuming the weighting factor is unity). At small time $t, F(q t u(L))$ dominates the decay of $G(q t, L)$, while $F\left(q t u\left(l_{d}\right)\right)$ is almost a constant. This is because the decay rate of $F(q t u(R))$ is proportional to $R^{1 / 3}$ according to the Kolmogorov theory [21]. At large time $t$, $F(q t u(L))$ has decayed to zero, and $F\left(q t u\left(l_{d}\right)\right)$ determines the decay of $G(q t, L)$.

Therefore, the initial decay of $G(q t, L)$ is dominated by the large-scale motions, and the small-scale motions only contribute to the long-time tail of $G(q t, L)$. In principle, one can obtain information about velocity fluctuations at all length scales $(0<R \leq L)$ from a single measurement of $G(q t, L)$. However, such an analysis requires a detailed modeling of the velocity-density function $P(V, R)$ in Eq. (2). The initial decay time $\tau(L)$ of $G(q t, L)$, on the other hand, can be extracted from the data without modeling the density function $P(V, R)$. As mentioned above, the decay time $\tau(L)$ is proportional to $[q u(L)]^{-1}$, where $u(L)$ is the characteristic velocity difference at the scale $L$. Turbulent motions at various length scales can be examined by measuring the $L$ dependence of the decay time $\tau(L)$.

It is often difficult to determine the initial decay time of a nonexponential decaying function. We therefore use the zeroth moment of $G(q t, L)$ as a definition of the decay time $\tau(L)$. With this definition, which emphasizes the initial decay of $G(q t, L), \tau(L)$ is

$$
\tau(L)=\int_{0}^{\infty} d t G(q t, L) .
$$

Using Eq. (7), one can numerically calculate $\tau(L)$ from the measured $G(q t, L)$ without knowing the exact analytic form of $G(q t, L)$. The advantages of using the HS technique are its high spatial resolution and ease of use. The high signal-to-noise ratio of the technique ensures that experimental errors are essentially statistical. The uncertainties for $\tau(L)$ in our measurements were below $5 \%$. At a moderately high scattering intensity ( $I \approx 10^{5}-10^{6}$ counts/s), it only took $\approx 5$ min to collect the data with an adequate signal-to-noise ratio when the slit width $L=2 \mathrm{~mm}$. At the smallest slit width $L=0.1 \mathrm{~mm}$, it was necessary to collect data for roughly $30 \mathrm{~min}$.

\section{EXPERIMENTAL RESULTS}

In order to map out the turbulent-velocity field and to observe the effect of the polymer on velocity gradients, more than a thousand correlation functions have been measured in different scattering geometries. The function $G(q t, L)$ was measured as a function of the angular velocity $\omega$ of the inner rotating cylinder $(\omega=2 \pi f$, with $f$ in turns per second), the slit width $L$, and the spatial position of the laser beam in the flow cell. It was established in I that the turbulence in our flow cell is neither homogeneous nor isotropic. Measurements of $G(q t, L)$ in water alone revealed that in our working range of $\omega$, the predominant turbulent-velocity gradient was in the horizontal $(r, \phi)$ plane, and the strongest velocity gradient in the horizontal plane was in the radial direction. We now discuss scaling properties of the turbulence in the PEO solution and the anisotropy of turbulent-drag reduction. Some results in this section have been briefly reported in Ref. [31]. 


\section{A. Scaling properties of the turbulence in polymer solutions}

Similar to the situation in water, the measured $G(q t, L)$ in the PEO solution is found to be of the scaling form as shown in Eq. (3). Log-log plots of $G(q t, L)$ for various values of the slit width $L$ and the angular velocity $\omega$, at the fixed scattering geometry and the fixed beam position in the flow cell, can be brought into coincidence by sliding them horizontally with respect to each other. The decay time $\tau(L)$ defined in Eq. (7) [see the discussion about $\tau(L)$ below] quantitatively characterizes the amount of the horizontal translation that is required to bring the correlation functions into coincidence. Figure 3 shows the typical $G(\kappa)$ as a function of $\kappa=t / \tau(L)$ for various values of $\omega$ and $L$. Since $\tau(L) \simeq[q u(L)]^{-1}$, where $u(L)$ is the characteristic velocity difference at the scale $L$, the scaling argument $\kappa$ can also be written as $\kappa=q t u(L)$. The correlation functions in Fig. 3 were measured in the 55ppm PEO solution at the A geometry. The scaling behavior of the measured $G(q t, L)$ is also found in other scattering geometries and beam positions.

Another important quantity in the statistical description of turbulence is the scaling velocity $u(R)$ as a function of the spatial separation $R$. The scale dependence of the scaling velocity can be examined by measuring the $L$ dependence of the decay time $\tau(L)$, since $\tau(L) \sim[q u(L)]^{-1}$. The decay time $\tau(L)$ in the polymer solution still obeys the power law $\tau(L) \sim L^{-\xi}$ [i.e., $\left.u(L) \sim L^{\xi}\right]$. Figure 4 compares the $L$ dependence of the measured $\tau(L)$ in water (lower curve) and in the 55-ppm PEO solution (upper curve). The measurements were made when $\omega=209 \mathrm{sec}^{-1}$ and at the $\mathrm{R}$ geometry. It is seen from Fig. 4 that $\log [\tau(L)]$ lies on a straight line when $L$ is in the range $0.2 \mathrm{~mm} \leq L \leq 1.4 \mathrm{~mm}$. The largest value of $L$ over which scaling is observed appears to be determined by the requirement that the light received by the photomultiplier be spatially coherent. This condition

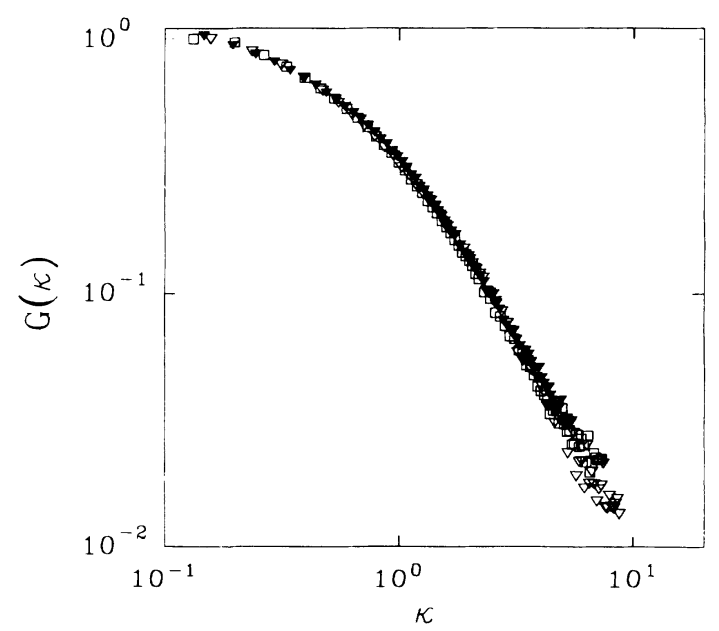

FIG. 3. The scaling function $G(\kappa)$ vs $\kappa=q t u(L) \simeq t / \tau(L)$ in the 55-ppm PEO solution at the A geometry. The experimental conditions are $L=1.0 \mathrm{~mm}, \omega=209 \mathrm{sec}^{-1}$ (open triangles); $L=0.2 \mathrm{~mm}, \omega=209 \mathrm{sec}^{-1}$ (solid triangles); and $L=0.3 \mathrm{~mm}$, $\omega=262 \sec ^{-1}$ (open squares).

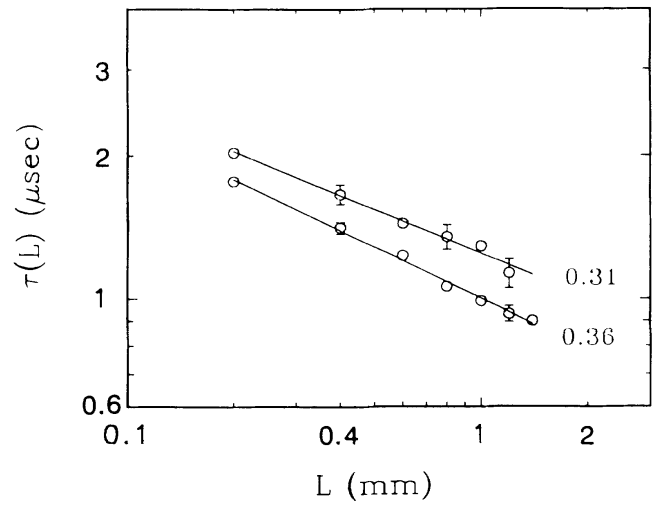

FIG. 4. Variations of the decay time $\tau(L)$ with the slit width $L$ measured in water (lower curve) and in the 55-ppm PEO solution (upper curve). The measurements were made when $\omega=209$ $\sec ^{-1}$ and at the $\mathbf{R}$ geometry. The solid lines are the power-law fits, and the number labeled beside a line is the slope of that line.

was satisfied in our experimental setup as long as $L$ was less than $2 \mathrm{~mm}$. The lower cutoff at small $L$ was controlled by the laser-beam diameter $(\simeq 0.1 \mathrm{~mm})$. The solid lines in Fig. 4 are the power-law fits to the data. The uncertainty for the slope $\xi$ is below $10 \%$ for water and $15 \%$ for the PEO solution.

The fitted value of $\xi$ for the PEO solution is 0.31 , which is slightly smaller than that for water $(\xi=0.36)$, but the difference is within experimental error. A larger error for the measured $\tau(L)$ in the PEO solution is due to the mechanical degradation of the polymer molecules in the solution. It is found that the viscosity of the $100-\mathrm{ppm}$ PEO solution is decreased from $1.16 \eta_{w}$ to $1.08 \eta_{w}$ after it was continuously stirred for an hour. Our turbulent-drag measurements show that when the PEO solution has been continuously stirred for $10 \mathrm{~min}$, the wall stress (force per unit area) on the inner rotating cylinder starts to increase. This mechanical degradation was also seen in other flow systems [32]. Figure 4 shows that the value of the exponent $\xi$ in the $\mathbf{R}$ geometry are close to the Kolmogorov value, indicating that the turbulence in the geometry is nearly fully developed. Though the exponents $\xi$ in the two fluids are almost the same, the absolute value of the decay time $\tau(L)$ is increased [ $u(L)$ is reduced] by a factor of 1.2 in the PEO solution (keeping the other conditions unchanged). This indicates a suppression of turbulentvelocity gradients by the polymer molecules. The suppression effect is seen for various slit widths from 0.2 to $1.4 \mathrm{~mm}$, and it is also observed in the A geometry. The above measurements of $G(q t, L)$ were performed in the midline (bulk region) of the gap between the two cylinders $(3.5 \mathrm{~cm}$ away from the outer wall).

As mentioned in Sec. II, the decay time $\tau(L)$ defined in Eq. (7) is only sensitive to the initial decay of $G(q t, L)$, and hence to the large-scale motions of the turbulent flow. The range of the scales is controlled by the slit width $L(0.2 \mathrm{~mm} \leq L \leq 1.4 \mathrm{~mm})$. Therefore, Figs. 3 and 4 suggest that scaling concepts can still be used to describe the turbulence in the polymer solution and that largescale velocity fluctuations in the PEO solution are suppressed compared with those in water. However, the 
statistical properties of these fluctuations over varying length scales remain unchanged (the scaling exponent $\xi$ is roughly the same as that in water). To see what happens in smaller scales, on which many theoretical efforts have focused, we now compare the functional form of $G(q t, L)$ in the PEO solution with that in water. Special attention is given to the long-time tail of $G(q t, L)$. This is because the initial decay of $G(q t, L)$ is dominated by the largescale motions, and the small-scale motions contribute to the long-time tail of $G(q t, L)$.

The scaling behavior for $G(q t, L)$ in the PEO solution was observed at various length scales and Reynolds numbers, with the spatial position of the laser beam and the scattering geometry fixed. When one compares $G(q t, L)$ measured in the PEO solution with those in water (keeping the other conditions unchanged), it is found that the functions $G(q t, L)$ do not scale mutually. Figure 5 compares the measured $G(\kappa)$ in the PEO solution (solid curve) and in water (open circles). The two correlation functions were measured at $L=1.0 \mathrm{~mm}, \omega=209 \mathrm{sec}^{-1}$, and in the $\mathbf{R}$ geometry. It is clearly seen that the two functions fail to scale with each other at large times, suggesting that the small-scale motions in the two fluids are very different. It is also shown in Fig. 5 that the function $G(\kappa)$ in the PEO solution decays slower at large $\kappa$ compared with that in water. Since $G(q t, L)$ is a superposition of decaying functions (decay modes), each of the decay modes characterizing the fluid motion at different length scales (see Sec. II), a slower decay of $G(q t, L)$ at large $t$ may indicate two different trends: either an increase in the number of decay modes at small scales or a suppression of small-scale motions, if the number of decay modes remains unchanged. The former indication is unlikely because the Reynolds number in the two fluids is the same. Notice that the number of active degrees of freedom per unit volume in a turbulent flow is proportional to $\mathrm{Re}^{9 / 4}$ (Ref. [33]). Therefore, Fig. 5 suggests that the small-scale motions in the polymer solution are strongly suppressed. This suppression effect is observed

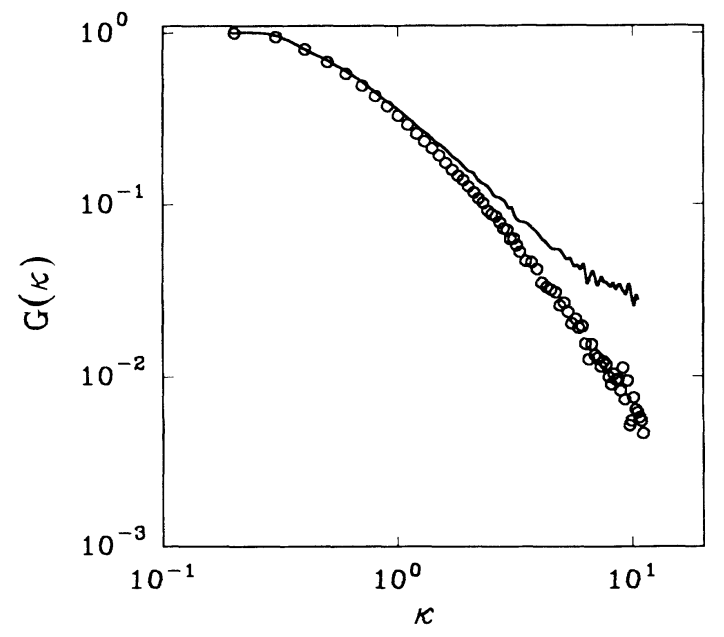

FIG. 5. The scaling function $G(\kappa)$ vs $\kappa=q t u(L) \simeq t / \tau(L)$ in water (open circles) and in the 55-ppm PEO solution (solid curve). The two correlation functions were measured when $\omega=209 \mathrm{sec}^{-1}, L=1.0 \mathrm{~mm}$, and in the R geometry. at various values of $L$ and $\omega$ and also in the A geometry.

Our experimental results in this section thus suggest that the addition of polymer to water causes a suppression of turbulent-velocity fluctuations over the whole spectrum of length scales. The large-scale fluctuations are suppressed, but their statistical properties remain unchanged (the scaling exponent $\xi$ is roughly the same). The small-scale fluctuations, on the other hand, are damped out much more strongly, resulting in a different functional form for the velocity-density function $P(V, R)$. The latter observation is consistent with the notion that the polymer-turbulence interaction causes a truncation of the turbulent-energy cascade at small scales $[2,5,6]$. The measurements are also in agreement with LDV results that in turbulent polymer solutions the lifetime of largescale vortices is increased and the high-frequency velocity fluctuations (small-scale motions) are reduced $[12,13]$.

\section{B. Anisotropy in turbulent-drag reduction}

The above measurements of $G(q t, L)$ show that the polymer molecules suppress the turbulent-velocity differences in the horizontal $(r, \phi)$ plane (A and $\mathrm{R}$ geometries). However, no suppression effect was observed when the incident beam is along the $z$ direction $\left(\mathrm{V}_{a}\right.$ geometry) for various $\omega$ up to $262 \mathrm{sec}^{-1}$; the measured $G(q t, L)$ in water and in the PEO solution are the same. This is because the fluid flow in the vertical direction is less turbulent as indicated by a slow decay of $\boldsymbol{G}(q t, L)$ and a small exponent $\xi[19]$. To generate more turbulence in this direction, we deliberately inserted four radial baffle plates placed symmetrically on the wall of the outer cylinder (see the $\mathbf{V}_{a}$ and $\mathbf{V}_{r}$ geometries in Fig. 1). The width of each baffle plate was $1.3 \mathrm{~cm}$, and its height was the same as that of the outer wall. This is a typical design for a mixing vessel [34].

In the baffled cell one can clearly see that in the $z$ direction there are large-scale velocity fluctuations, which are absent in the unbaffled cell. When the fluid hits the corner where the outer wall and a baffle plate meet, the flow direction is forced to change either in the horizontal plane (where the fluid element has to be stretched) or in the vertical direction (up-down motion). The vertical motion was observed by eye through the addition of polymeric flakes (Kalliroscope AQ1000, $1 \%$ in concentration) to water. The measured $G(q t, L)$ shows that in the baffled cell the velocity gradient in the $r$ direction is reduced. Thus the insertion of the baffle plates enhances velocity fluctuations in the $z$ direction and reduces the velocity gradient in the $r$ direction. One may view the turbulence in the baffled cell as a turbulent wake generated by the baffle plates. More detailed discussions on the turbulent velocity field can be found in I.

We now discuss the scaling properties of $G(q t, L)$ for the PEO solution in the baffled cell. Similar to the situation in the unbaffled cell, the measured $G(q t, L)$ in the baffled cell is found to be of the scaling form as shown in Eq. (3). $L o g-\log$ plots of $G(q t, L)$ for various values of $L$ and $\omega$, given the scattering geometry and the beam position in the flow cell, can be superimposed by scaling the time axis to $\kappa=t / \tau(L)$. Figure 6 shows the typical $G(\kappa)$ as a function of $\kappa=q t u(L) \simeq t / \tau(L)$ for various values of 


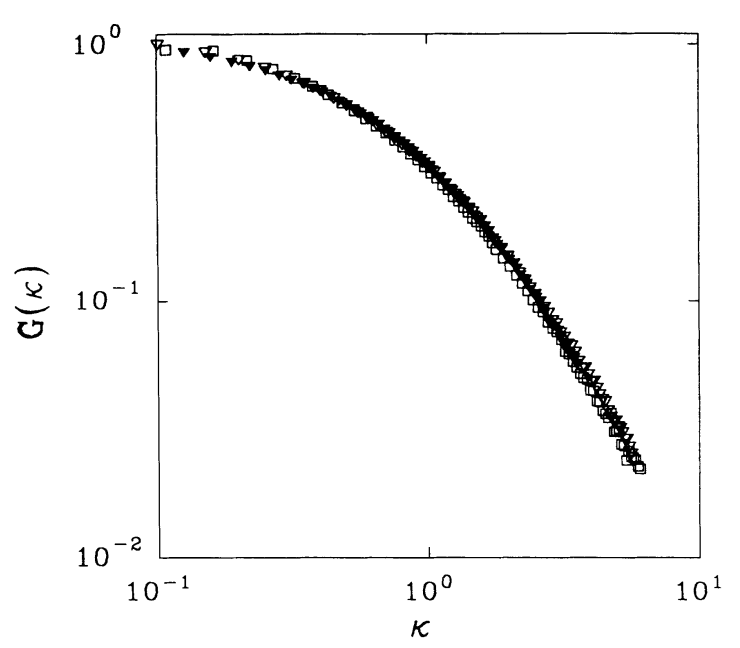

FIG. 6. The scaling function $G(\kappa)$ vs $\kappa=q t u(L) \simeq t / \tau(L)$ for the 55-ppm PEO solution in the baffled cell at the A geometry. The experimental conditions are $L=1.0 \mathrm{~mm}, \omega=209 \mathrm{sec}^{-1}$ (open triangles); $L=0.2 \mathrm{~mm}, \omega=209 \mathrm{sec}^{-1}$ (solid triangles); and $L=0.5 \mathrm{~mm}, \omega=262 \mathrm{sec}^{-1}$ (open squares).

$\omega$ and $L$ in the 55-ppm PEO solution. These correlation functions were measured in the baffled cell and at the $A$ geometry. The decay time $\tau(L)$ in the baffled cell still obeys the power law $\tau(L) \sim L^{-\xi}$, and the values of the exponent $\xi$ in the $A$ and $V_{a}$ geometries are roughly the same as those measured in the unbaffled cell [19]. The above results suggest that scaling concepts can also be used to describe the turbulent polymer solution in the baffled cell.

Perhaps the most dramatic effect occurring in the baffled cell is the suppression of velocity differences by addition of the PEO polymer in the $\mathrm{V}_{a}$ geometry. This suggests that the polymer molecules suppress the vertical velocity fluctuations produced by the baffle plates. The upper two curves in Fig. 7 compare the measured $\boldsymbol{G}(q t, L)$ in water (open circles) and in the PEO solution (solid circles). The measurements were performed when $\omega=203 \mathrm{sec}^{-1}, L=0.5 \mathrm{~mm}$, and in the $\mathrm{V}_{a}$ geometry. The position of the vertical beam was $1.5 \mathrm{~cm}$ away from the edge of a baffle plate in the downstream direction and 1.3 $\mathrm{cm}$ away from the outer wall (see the $\mathrm{V}_{a}$ geometry in Fig. 1). The decay time $\tau$ in the PEO solution is increased by a factor of 1.4. This suppression effect is seen for several slit widths from 0.2 to $1.4 \mathrm{~mm}$. The difference in the measured $G(q t, L)$ between water and the PEO solution gradually diminishes when the angular velocity is reduced. It is observed that the two curves of $G(q t, L)$ coincide when $\omega=94 \mathrm{sec}^{-1}$. The maximum suppression effect in this scattering geometry occurs in the near-baffle region $(\sim 1.3 \mathrm{~cm}$ away from the outer wall in the downstream direction) where the vertical velocity fluctuations are the strongest. When the incident beam is moved to the region close to the inner rotating cylinder, the vertical velocity fluctuations are reduced, and so is the suppression effect.

When the scattering geometry is changed to $\mathrm{V}_{r}$, the measured $G(q t, L)$ in water and in the PEO solution

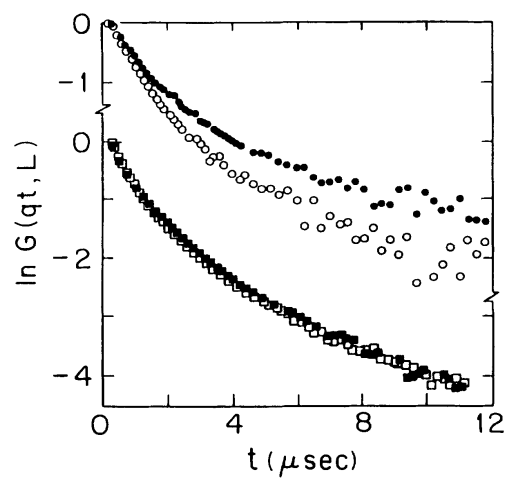

FIG. 7. The measured $G(q t, L)$ in the baffled cell for water (open symbols) and for the 55-ppm PEO solution (solid symbols) when $\omega=203 \mathrm{sec}^{-1}$ and $L=0.5 \mathrm{~mm}$. The upper two curves were measured in the $\mathrm{V}_{a}$ geometry, and the lower two curves were obtained in the $\mathrm{V}_{r}$ geometry.

remain the same for various $L$ and $\omega$. This is shown by the lower two curves in Fig. $7\left(\omega=203 \mathrm{sec}^{-1}, L=0.5\right.$ $\mathrm{mm}$; see the $\mathrm{V}_{r}$ geometry in Fig 1). We notice that the measured $G(q t, L)$ in water for the $\mathrm{V}_{a}$ geometry decays twice as fast as that for the $V_{r}$ geometry when $\omega=203$ $\sec ^{-1}$ and $L=1.0 \mathrm{~mm}$. The data in Fig. 7 thus reveal a large anisotropic suppression of turbulent-velocity differences in the polymer solution. In particular, as shown in Fig. 7, polymer molecules suppress the velocity difference $\left(\partial v_{\phi} / \partial z\right) L$, but not the other two components listed under the $\mathbf{V}_{a}$ and $\mathbf{V}_{r}$ geometries in Fig. 1 .

A slightly larger suppression effect is also observed in the baffled cell (compared with that in the unbaffled cell) when the laser beam enters the cell along the $\phi$ direction (the A geometry) at $\omega=209 \mathrm{sec}^{-1}$. The larger effect in the baffled cell indicates that the turbulent-velocity gradient in the cell is enhanced in the mean flow direction. The difference in $G(q t, L)$ between water and the PEO solution disappeared when the scattering geometry was changed to $R$. This can be understood by the fact that the turbulent-velocity gradients in the radial direction are reduced in the baffled cell [19]. The measurements of $G(q t, L)$ in the PEO solution, therefore, show that the predominant polymer effect is on the velocity component along the mean flow direction. Gradients of this component appear to be suppressed in all directions in which the gradients are substantial. This effect presumably arises from the elastic stress in the polymer chains opposing the stretching and shearing of fluid elements.

The observed suppression of velocity differences in the PEO solution is found to be associated with a reduction of turbulent drag. The turbulent drag was determined by measuring the wall stress $T_{w}$ (force per unit area) on the inner rotating cylinder in both the unbaffled cell and the baffled cell. Figure 8 (a) shows the measured $T_{w}$ in the unbaffled cell as a function of the angular velocity $\omega$ for three solutions: distilled water (solid circles), the 25wt \% glycerol aqueous solution (open circles), and the 55-ppm PEO aqueous solution (diamonds). An onset of the drag reduction appears to occur at $\omega_{0} \simeq 250 \mathrm{sec}^{-1}$. It can be seen that the drag reduction increases with increasing $\omega$. For the highest accessible angular velocity 
$\omega=385 \mathrm{sec}^{-1}$, a $10 \%$ drag reduction was achieved. The $25 \%$ glycerol solution $\left(\eta=2.08 \eta_{w}\right)$ was used to assure that the drag reduction in the polymer solution was not simply the result of its increased viscosity $\left(\eta=1.08 \eta_{w}\right)$ relative to water.

According to turbulent boundary layer theory for a simple fluid, the wall stress $T_{w}$ has the following form [35]:

$$
T_{w} \sim \rho U^{2}\left(\operatorname{Re}_{a}\right)^{-\alpha} \sim \rho^{1-\alpha} \eta^{\alpha} U^{2-\alpha} a^{-\alpha} \equiv A \omega^{2-\alpha},
$$

where $\rho$ is the density of the fluid, $a$ is the radius of the inner rotating cylinder, and $U=a \omega$ is the characteristic velocity. The Reynolds number $\operatorname{Re}_{a}$ is defined as $\operatorname{Re}_{a}=\omega a^{2} / v$, where $v$ is the kinematic viscosity of the fluid. A typical value of $\alpha$ is 0.2 . Equation (8) is an empirical scaling law, which holds for the turbulent wall stress on many smooth surfaces, such as a flat plate, a rotating disk, and a straight pipe. Our measured $T_{w}$ for distilled water and the 25 wt. \% glycerol solution $\left(\eta=2.08 \eta_{w}\right)$ are found to be well fitted to Eq. (8) with $\alpha=0.2$ [the solid curves in Fig. 8(a)]. Figure 8(b) replots the data in Fig. 8(a) for distilled water (solid circles) and the $25 \mathrm{wt}$. \% glycerol aqueous solution (open circles) as a function of $\omega^{1.8}$. The solid lines are the linear fits to data,
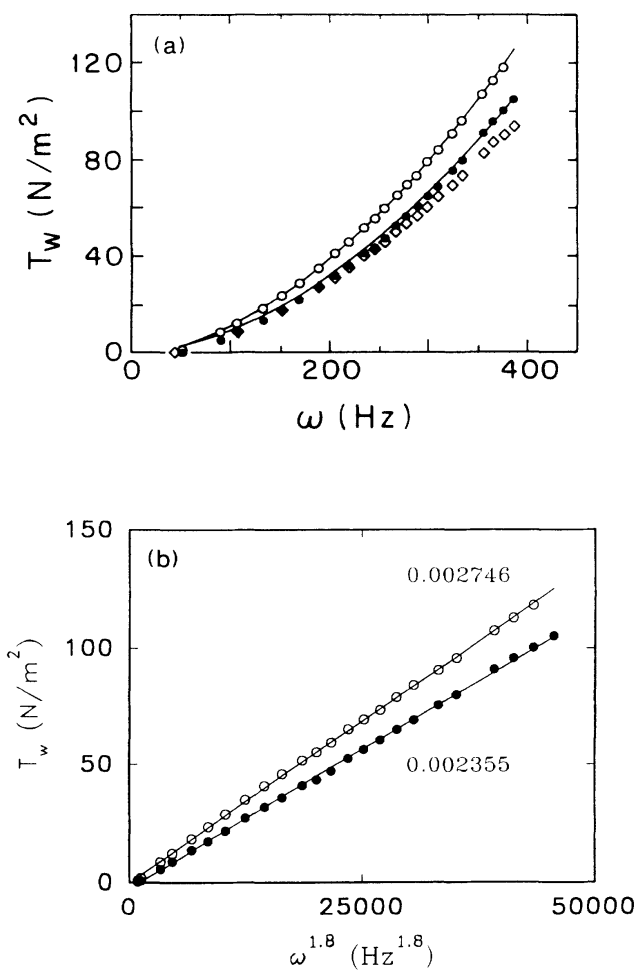

FIG. 8. (a) The measured wall stress $T_{w}$ in the unbaffled cell as a function of $\omega$ for three solutions: distilled water (solid circles), the $25 \mathrm{wt}$. \% glycerol aqueous solution (open circles), and the 55-ppm PEO solution (diamonds). The solid curves are the fits to Eq. (8) with $\alpha=0.2$. In (b) we replot the data for distilled water (solid circles) and the $25 \mathrm{wt}$ \% glycerol aqueous solution (open circles) as a function of $\omega^{1.8}$. The solid lines are the linear fits to data, and the numbers on the graph are the slopes of the fitted straight lines. and the numbers on the graph are the slopes of the fitted straight lines. It can be seen that the two fitted straight lines have different slopes, and the ratio of the two slopes is as follows:

$$
\beta=A_{\text {gly }} / A_{\text {water }}=1.17 .
$$

From Eq. (8) one expects the ratio

$$
\beta=\left(\rho / \rho_{w}\right)^{0.8}\left(\eta / \eta_{w}\right)^{0.2}=1.20,
$$

which agrees well with our drag measurement.

Figure 9 shows the measured $T_{w}$ in the baffled cell as a function of $\omega$ for distilled water (closed circles) and the PEO aqueous solution with $c=55 \mathrm{ppm}$ (diamonds). One can clearly see an enhancement of the drag reduction at large $\omega$. For the largest accessible $\omega\left(=310 \mathrm{sec}^{-1}\right)$, we obtain a $25 \%$ drag reduction, which is 2.5 times larger than in the unbaffled cell. Another important change is that in the baffled cell the onset angular velocity $\omega_{0}$ is greatly reduced from $\omega_{0} \simeq 250 \mathrm{sec}^{-1}$ to $\omega_{0} \simeq 150 \mathrm{sec}^{-1}$. It is also found that the measured $T_{w}$ remains the same value for the PEO solution with $c=100 \mathrm{ppm}$, which indicates that the drag reduction is already saturated in our concentration range [1]. It is notable that $T_{w}$ in the baffled cell is 1.55 times larger than that in the unbaffled cell, so the flow field is clearly changed by the baffles. Yet the exponent $\alpha$ in Eq. (8) has the same value for both cells filled with water or the glycerol solution. However, for the PEO solution the exponent $\alpha$ changes its value to $\alpha=0.4$ in the baffled cell.

\section{An interesting wall effect}

The above measurements of $G(q t, L)$ for the $\mathrm{V}_{a}$ and $\mathrm{V}_{r}$ geometries in the baffled cell were performed in the bulk region of the turbulent fluid and are little affected by changing the direction of the rotation of the inner cylinder. However, when the vertical incident beam is deliberately moved to the corner where a baffle plate and the outer wall meet, it is found that the polymer additive affects the upstream flow differently from the downstream flow. Figure 10 shows the measured $G(q t, L)$ in water (solid circles) when $\omega=94 \mathrm{sec}^{-1}, L=1.5 \mathrm{~mm}$, and $\mathbf{q}=\mathbf{q v}_{\mathrm{v}_{r}}$ [see Eq. (6)]. The incident beam was $1.5 \mathrm{~cm}$ away from a baffle plate in the $\phi$ direction (upstream side) and

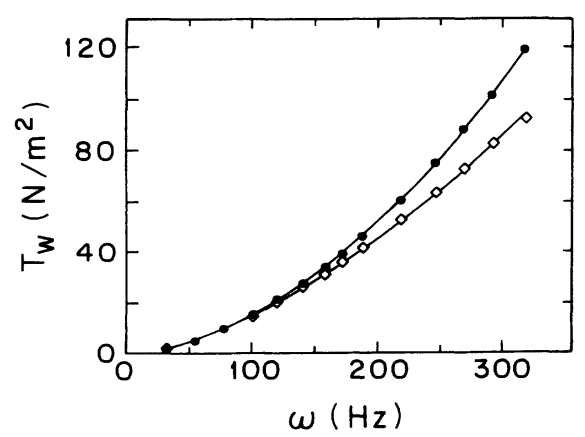

FIG. 9. The measured wall stress $T_{w}$ in the baffled cell as a function of $\omega$ for distilled water (solid circles) and the 55-ppm PEO solution (diamonds). The upper solid curve is a fit to Eq. (8) with $\alpha=0.2$, and the lower solid curve is the fit with $\alpha=0.4$. 


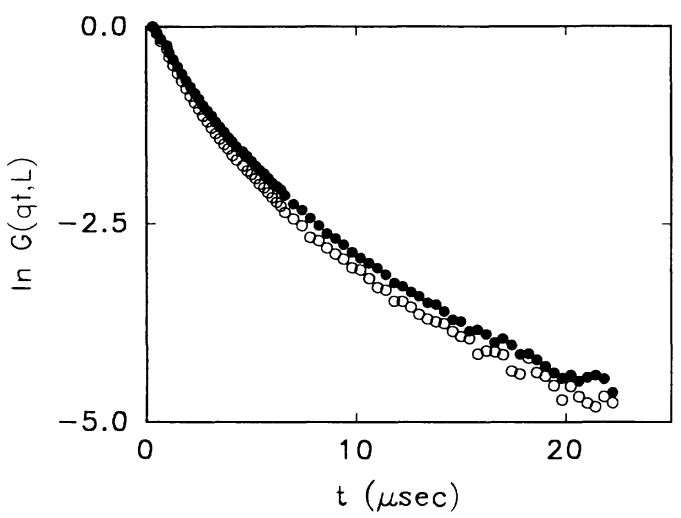

FIG. 10. The measured $G(q t, L)$ in water (solid circles) and in the PEO solution (open circles). The two measurements were made in the $V_{r}$ geometry when $\omega=94 \mathrm{sec}^{-1}$ and $L=1.5 \mathrm{~mm}$. The incident beam was $1.5 \mathrm{~cm}$ away from a baffle plate in the $\phi$ direction (upstream side) and was $4 \mathrm{~mm}$ away from the outer wall.

was $4 \mathrm{~mm}$ away from the outer wall. The open circles in Fig. 14 show measurements made under the same conditions, but with water replaced by the 50-ppm PEO solution. It is clearly seen that $G(q t, L)$ in the PEO solution decays faster than that in water, indicating an enhancement of velocity differences. The decay time $\tau$ for the PEO solution is reduced by a factor of 1.12 . The maximum enhancement was found at small $\omega\left(\omega=94 \mathrm{sec}^{-1}\right)$, and at large $\omega\left(\omega=203 \mathrm{sec}^{-1}\right)$ the difference in $G(q t, L)$ between water and the PEO solution disappeared. When the incident laser beam was changed to the downstream side of the baffle plate (by changing the direction of the rotation), no enhancement of velocity differences was found in the PEO solution. Instead, the measured $G(q t, L)$ in the PEO solution shows an expected slower decay than that in water, i.e., velocity differences are suppressed. The suppression effect is even larger than that shown in Fig. 7. The measured $\tau$ in the PEO solution is increased by a factor of 1.13 when $\omega=94 \mathrm{sec}^{-1}$, and it is increased by a factor of 1.32 when $\omega=203 \mathrm{sec}^{-1}$.

It has been known [36] that there is an enhancement of the recirculating vortex formation in the upstream corner of a contraction flow for concentrated polymer solutions and polymer melts. At low Reynolds numbers the vortex grows with increasing flow rate, and at higher Reynolds numbers when the inertia becomes important, the size of the vortex decreases with increasing flow rate. It is possible that the enhancement of velocity differences observed in our dilute polymer solution at low Reynolds number is related to the enhancement of the vortex formation. A systematic study of velocity differences in the baffled cell at low Reynolds numbers may be of interest in itself.

\section{DISCUSSION}

As mentioned in Sec. III B, the observed anisotropic suppression of velocity differences in the mean flow direction may result from the elastic stress in the polymer chains opposing the stretching and shearing of fluid elements. To judge the effect of the turbulent elongational shear $S(\mathbf{r})$ on polymer chains, one has to compare $S(\mathbf{r})$ with the polymer relaxation rate $\Gamma=\left(k_{B} T / 6 \pi \eta_{w}\right) R_{h}^{-3}$. When $S(\mathbf{r})$ is small compared to $\Gamma$, polymer molecules relax in a time shorter than $S(\mathbf{r})^{-1}$, and hence the stretching effect on the polymer molecules will be small (weak-shear region). In the strong-shear region, where $S(\mathbf{r})>\Gamma$, the shear will strongly stretch the polymer chains, and this stretching is believed responsible for the onset of the drag reduction.

In our measurements the angular velocity $\omega$ ranged from 42 to $385 \mathrm{sec}^{-1}$. The corresponding Reynolds number $\mathrm{Re}_{a}=\omega a^{2} / v$ is in the range $3.3 \times 10^{4}$ to $3 \times 10^{5}$, where $a(=2.8 \mathrm{~cm})$ is the radius of the inner rotating cylinder. Our visual observations show that the flow in the unbaffled cell is turbulent even at the lowest angular velocity $\omega=42 \mathrm{sec}^{-1}$. The Reynolds number corresponding to this angular velocity $\left(\operatorname{Re} \simeq 10^{4}\right)$ is much larger than the critical Reynolds number $\mathbf{R e}_{c}$ for the Taylor vortex instability $\left(\operatorname{Re}_{c} \simeq 10^{2}\right)$ [37]. To have an order-ofmagnitude estimate for the onset of the drag reduction, we invoke the Kolmogorov theory [21] of fully developed turbulence and the turbulent boundary layer theory [35] because no other theory is available for flows with the moderate Reynolds number. The turbulent elongational shear $S(\mathbf{r})$ is estimated using the maximum turbulent shear [21] $S\left(l_{d}\right) \simeq v \mathrm{Re}^{3 / 2} / a^{2}$, which is the most important shear for stretching polymer chains [6]. Here $l_{d} \simeq l_{0} \mathrm{Re}^{-3 / 4}$ is the Kolmogorov dissipation length.

In analyzing turbulence near a wall, one should use a Reynolds number $\mathrm{Re}_{\delta}$, which is based on the turbulent boundary layer thickness $\delta$ rather than $\operatorname{Re}_{a}$. The two Reynolds numbers can be related by the empirical equation [35]

$$
\operatorname{Re}_{\delta}=0.37 \operatorname{Re}_{a}^{4 / 5}
$$

Now we can estimate the onset of the drag reduction in terms of a critical Reynolds number $R_{c}$ by equating $S\left(l_{d}\right)$ to $\Gamma$. This also defines an onset angular velocity $\omega_{0}$ using $R_{c}=\omega_{0} a^{2} / v$. For our polymer with $R_{h} \simeq 80 \mathrm{~nm}$, we obtain the onset angular velocity $\omega_{0} \simeq 230 \mathrm{sec}^{-1}$, at which $S\left(l_{d}\right) \simeq 500 \mathrm{sec}^{-1}$. At this Reynolds number the Kolmogorov dissipation length $l_{d} \simeq 0.04 \mathrm{~mm}$. From Fig. 8(a) one can see that the above estimation for the onset of the drag reduction agrees satisfactorily with the measurement.

We now address the most difficult question: how the stretched polymer chains interact with hydrodynamic flows at small scales. This question cannot be answered conclusively at present because of the following reasons. First of all, most theoretical models for turbulent-drag reduction have not yet led to any quantitative predictions that could be compared with experimental results. Secondly, because most experimental studies on turbulent-drag reduction have been focused on the hydrodynamic effect of polymer additives, microscopic information about stretching and orientation of polymer molecules in turbulent flows is not available. Nevertheless, our experimental results can still shed light on the theoretical modeling of turbulent-drag reduction. Measurements of the correlation function $G(q t, L)$ indicate that the suppression of velocity differences at small scales 
occurs in the bulk region of the turbulence. This is consistent with the Tabor-de Gennes approach [6], which discussed the properties of homogeneous, isotropic, three-dimensional turbulence in the presence of polymer additives without any wall effect. Their main idea is that the polymer effects on the turbulence at small scales are described by an elastic modulus, but not a viscosity.

Another finding of the experiment is that the suppression of turbulent-velocity differences occurs at both large scales and small scales. This suggests a coupling of velocity fluctuations among various scales, which is absent in the hypothesis that stretched polymer chains only affect the turbulent-energy cascade at small scales $\left(\sim l_{d}\right)$ via either the enhancement of the elongational viscosity $[2,5]$ or the elastic modulus of the stretched chains [6]. Landahl $[3,7,8]$ has discussed such a coupling and proposed that the major dynamic effect of polymer molecules is to cause stabilization of small-scale secondary motions in the viscous boundary layer, thereby inhibiting the production of turbulent bursts. The reduction of the turbulent bursts results in a turbulent-drag reduction. Changes of the larger-scale structures in the turbulent bulk region, due to intermittent bursts from viscous boundary layers, may be a dominant feature for the transport of momentum in turbulent flows. Each burst is an explosive production of Reynolds stress, and the HS technique is capable of measuring the velocity differences within the bursts. A main feature of the Landahl stability analysis is that the viscoelasticity does not seem to be the key fluid property; rather, the anisotropic stress caused by the stretching of the polymer chains in a turbulent flow is a more likely candidate. This conclusion is consistent with our finding that there exists a large anisotropic suppression of turbulent-velocity differences in the polymer solution.

Recently, Ryskin [5] calculated the viscosity increase in Lumley's model [2] using a raveling-unraveling polymer model ("yo-yo model") to describe the polymer stretching [5]. The viscosity increase was then related to some measurable drag-reduction parameters. Our turbulent-drag measurements show that the wall stress $T_{w}$ for the PEO solution in the baffled cell goes as $\omega^{1.6}$ ( $\alpha=0.4$ ), while $T_{w}$ measured in water is proportional to $\omega^{1.8}(\alpha=0.2$; see Fig. 9). This finding cannot be explained by an enhancement of the turbulent viscosity due to the stretching of polymer chains, as has been proposed $[2,5]$. It is believed that polymer molecules are stretched only in the turbulent bulk region where the viscosity is increased to a larger value $\eta_{t}$ relative to the solvent. In the viscous boundary layer, on the other hand, the laminar shear cannot stretch the polymer chains much, and hence the viscosity remains the same as $\eta_{\text {sol }}$, the viscosity of the solvent [2]. If indeed this is so, one would anticipate that the enhancement of the turbulent viscosity can only cause the amplitude $A$ in Eq. (8) to change, but not the exponent $\alpha$, as we now argue. If we replace the polymer solution by a simple fluid with the viscosity $\eta_{t}$, the resulting wall stress $T_{t}$ for the fluid will satisfy Eq. (8):

$$
T_{t} / T_{\text {sol }}=\left(\eta_{t} / \eta_{\text {sol }}\right)^{\alpha},
$$

where $T_{\text {sol }}$ is the wall stress for the solvent. The wall stress $T_{\text {PEO }}$ for the polymer solution is different from $T_{t}$ because the viscosity in the viscous boundary layer is different. The wall stress $T_{\text {PEO }}$ can be related to $T_{t}$ by the proposed equation [5] $T_{\mathrm{PEO}}=\left(\eta_{\mathrm{sol}} / \eta_{t}\right) T_{t}$. Therefore, the ratio of the two wall stresses between the polymer solution and its solvent is

$$
\beta=T_{\mathrm{PEO}} / T_{\mathrm{sol}}=\left(\eta_{\mathrm{sol}} / \eta_{t}\right)^{1-\alpha} .
$$

This suggests that the $\omega$ dependence of $T_{\text {PEO }}$ should be the same as that for the solvent, and this is inconsistent with our measurements.

It should be mentioned that the weak viscosity $\left(\eta^{0.2}\right)$ dependence of the measured $T_{w}$ in simple fluids [see Fig. $8(\mathrm{~b})$ ] indicates that there exists a viscous boundary layer near the wall of the rotating cylinder. From Eq. (8) one can see that the $\eta^{0.2}$ dependence is associated with an $\omega^{1.8}$ dependence of the wall stress $T_{w}$. When the inner rotating cylinder is replaced by a four-blade propeller (its diameter was $6.35 \mathrm{~cm}$, and the width of the blade was 1.27 $\mathrm{cm}$ ), the measured drag in the baffled cell goes as $\omega^{2}$ for both the PEO solution and water; no drag reduction is observed. This is because the drag for the propeller is mainly from the pressure difference across the blade (the pressure drag), while the friction drag (the skin drag) is very small.

\section{CONCLUSION}

We have studied turbulent-drag reduction in dilute PEO aqueous solution. The technique of photoncorrelation homodyne spectroscopy was used to measure turbulent-velocity differences in a concentric cylinder cell, in which the inner cylinder rotates. Different components of the velocity differences were probed by changing the direction of the scattering vector $q$. Velocity differences at various length scales were measured by varying the size of the scattering volume. A large anisotropic suppression of turbulent-velocity differences is found in the bulk region of the turbulent fluid. The suppression effect is associated with an enhancement of the turbulent-drag reduction. The experiment reveals that the predominant polymer effect is on the velocity component along the mean flow direction. Gradients of this component appear to be suppressed in all directions in which the gradients are substantial. This effect presumably arises from the elastic stress in the polymer chain opposing the stretching and shearing of fluid elements.

The measured correlation function $G(q t, L)$ is found to be of the scaling form $G(q t u(L))$ with $u(L) \sim L^{\xi}$, where $u(L)$ is the characteristic turbulent velocity at the length scale $L$. The log-log plots of $G(q t, L)$ for various values of the slit width $L$ and the angular velocity $\omega$, at the fixed scattering geometry and the fixed beam position in the flow cell, can be brought into coincidence by sliding them horizontally with respect to each other. The scaling behavior of $G(q t, L)$ is independent of the scattering geometries and the flow cells. However, when one compares the correlation functions measured in water with those in the PEO solution (keeping the other conditions unchanged), it is found that the above scaling description 
for $G(q t, L)$ is no longer valid. A scaling $G(q t u(L))$ indicates that the velocity-density function $P(V, R)$ has a scaling form $Q(V / u(R)) / u(R)$. The above results, thus, suggest that the scaling argument an still be used to describe the turbulence in the polymer solution.

By comparing the measurements in the PEO solution with those in water, we find that the polymer additive causes suppressions of velocity differences over the whole spectrum of length scales. The large-scale velocity fluctuations are suppressed, but their statistical properties over varying length scales remain unchanged by addition of the polymer. On the other hand, the small-scale fluctuations are damped out much more strongly, resulting in a different functional form for $P(V, R)$. The latter observation is consistent with the notion that the polymer- turbulence interaction causes a truncation of the turbulent-energy cascade at small scales. The measurements are also in agreement with LDV results that in turbulent polymer solutions the lifetime of large-scale vortices is increased, and the high-frequency velocity fluctuations (small-scale motions) are reduced. Still lacking is a theory providing quantitative predictions that can be used to compare with experimental results.

\section{ACKNOWLEDGMENTS}

We are indebted to T. A. Witten for stimulating comments and for the insights he has provided. This work was supported in part by the National Science Foundation under Grant No. DMR-8914351.
[1] P. S. Virk, AIChE J. 21, 625 (1975).

[2] J. L. Lumley, Annu. Rev. Fluid Mech. 1, 367 (1969); J. Polym. Sci. Macromol. Rev. 7, 263 (1973).

[3] J. L. Lumley and I. Kubo, in The Influence of Polymer Additives on Velocity and Temperature Fields, edited by B. Gampert (Springer, Berlin, 1984), p. 3.

[4] W.-M. Kulicke, M. Kotter, and H. Grager, Adv. Polym. Sci. 89, 1 (1989).

[5] G. Ryskin, Phys. Rev. Lett. 59, 2059 (1987); J. Fluid Mech. 178, 423 (1987).

[6] M. Tabor and P. G. de Gennes, Europhys. Lett. 2 (7), 519 (1986); P. G. deGennes, Physica A (Amsterdam) 140, 9 (1986).

[7] M. T. Landahl, in Proceedings of the Thirteenth International Congress on Theoretical and Applied Mechanics, Moscow, edited by E. Becker and G. K. Mikhoilov (Springer, Berlin, 1973), p. 177.

[8] M. T. Landahl, Phys. Fluids 20, S55 (1977).

[9] L. E. Drain, The Laser Doppler Technique (Wiley, New York, 1980).

[10] F. Durst, A Melling, and J. H. Whitelaw, Principles and Practice of Laser-Doppler Anemometry, 2nd ed. (Academic, New York, 1981).

[11] T. S. Luchik and W. G. Tiederman, J. Fluid Mech. 190, 241 (1988).

[12] M. Kwade, Rheol. Acta. 21, 120 (1982).

[13] R. Scharf, Rheol. Acta. 24,385 (1985).

[14] See, e.g., H. Tennekes and J. L. Lumley, A First Course in Turbulence (MIT Press, Cambridge, MA, 1972).

[15] B. J. Berne and R. Pecora, Dynamic Light Scattering (Wiley, New York, 1976).

[16] P. Tong, W. I. Goldburg, C. K. Chan, and A. Sirivat, Phys. Rev. A 37, 2125 (1988).

[17] A. Onuki, Phys. Lett. A 127, 143 (1988).

[18] P. J. Burke et al., J. Phys. A 3, 216 (1970).

[19] P. Tong, W. I. Goldburg, and J. S. Huang, preceding paper, Phys.Rev. A 45, 7222 (1992).

[20] C. W. van Atta and J. Park, in Statistical Models and Turbulence, edited by M. Rosenblatt and C. W. van Atta, Lec- ture Notes in Physics Vol. 12 (Springer, Berlin, 1972), p. 402.

[21] A. N. Kolmogorov, C. R. Dokl. Acad. Sci. URSS. 30, 301 (1941); 31, 538 (1941).

[22] U. Frisch, P. Sulem, and M. Nelkin, J. Fluid Mech. 87, 719 (1978).

[23] U. Firsch, in Turbulence and Predictability in Geophysical Fluid Dynamics and Climate Dynamics, edited by M. Ghil, R. Benzi, and G. Parisi (North-Holland, Amsterdam, 1985), p. 71.

[24] P. Tong and W. I. Goldburg, Phys. Fluids 31, 2841 (1988); Phys. Lett. A 127, 147 (1988); W. I. Goldburg and P. Tong, Phys. Scr. 40, 424 (1989).

[25] W. I. Goldburg, P. Tong, and H. K. Pak, Physica D 38, 134 (1989); H. K. Pak, W. I. Goldburg, and A. Sirivat, Fluid Dynamics Res. 8, 19 (1991).

[26] B. Knight and L. Sirovich, Phys. Rev. Lett. 65, 1356 (1990).

[27] See, e.g., L. E. Drain, The Laser Doppler Technique (Ref. [9]), p. 183.

[28] Union Carbide, Information Manual, 1988 (unpublished).

[29] Polymer Handbook, 3rd ed., edited by J. Brandrup and E. H. Immergut (Wiley, New York, 1989), pp. VII/22 and VII/41.

[30] A. Onuki, Prog. Theor. Phys. S99, 382 (1989).

[31] P. Tong, W. I. Goldburg, J. S. Haung, and T. A. Witten, Phys. Rev. Lett. 65, 2780 (1990).

[32] G. E. Gadd, Nature (London) 206, 463 (1965).

[33] L. D. Landau and E. M. Lifshitz, Fluid Mechanics (Pergamon, London, 1959).

[34] S. Nagata, Mixing: Principles and Applications (Wiley, New York, 1975).

[35] H. Schlichting, Boundary-Layer Theory, 7th ed. (McGraw-Hill, New York, 1979).

[36] D. V. Boger, Annu. Rev. Fluid Mech. 19, 157 (1987).

[37] R. C. DiPrima and H. L. Swinney, in Hydrodynamic Instabilites and the Transition to Turbulence, edited by $\mathrm{H}$. L. Swinney and J. P. Gollub (Springer, Berlin, 1985). 